\title{
Determining the neutron star equation of state using the narrow-band gravitational wave detector Schenberg
}

\author{
Guilherme F Marranghello and José C $N$ de Araujo
}

Instituto Nacional de Pesquisas Espaciais - Divisão de Astrofísica
Av. dos Astronautas 1758, São José dos Campos, 12227-010 SP,
Brazil

\begin{abstract}
We briefly review the properties of quasi-normal modes of neutron stars and black holes. We analyze the consequences of a possible detection of such modes via the gravitational waves associated with them, specially addressing our study to the Brazilian spherical antenna, on which a possible detection would occur at $3.0-3.4 \mathrm{kHz}$. A question related to any putative gravitational wave detection concerns the source that produces it. We argue that, since the characteristic damping times for the gravitational waves of neutron stars and black holes are different, a detection can distinguish between them; and also distinguish the neutron star's oscillating modes. Moreover, since the source can be identified by its characteristic damping time, we are able to extract information about the neutron star or black hole. This information would lead, for example, to a strong constrain in the nuclear matter equation of state, namely, the compression modulus should be $K \approx 220 \mathrm{MeV}$.
\end{abstract}

04.30.Db, 04.80.Nn, 95.55.Ym, 97.60.Jd, 97.60.Lf

\section{Introduction}

A new window to the Universe is about to be opened. With the detection of gravitational waves (GWs), astrophysicists expect to have the answer to many questions, as well as new ones. There are many detectors all around the world and, soon, even above us. With interferometric or resonant mass detectors, at low and high frequencies, we shall be able to see waves 
coming from coalescing binary systems, from a cosmological background, from catastrophic events etc.

Among the most promising sources of GWs, neutron stars (NSs) and black holes $(\mathrm{BHs})$ are the best candidates for a detection. These objects emit waves in a very wide spectrum of frequencies determined by their quasinormal modes of oscillation; and many works have been recently done to determine their characteristics (see Ref. 1] for a review).

We take a special attention to a small region of this spectrum, localized at 2.8-3.4 kHz, which is the region of operation of the resonant spherical antennas Mario Schenberg (Brazilian, 3.0-3.4 kHz) [2] and Mini-GRAIL (Dutch, 2.8-3.0 kHz) [3]. Both antennas are composed of $\sim 1$ ton and $\sim 70 \mathrm{~cm}$ diameter $\mathrm{CuAl} 6 \%$ spheres and will be operating in the frequency range mentioned above.

It is worth stressing that the identification of the direction of a source, making use of only one detector, is possible only if it is a spherical antenna [2. Several interferometer and bar detectors would be necessary to do the same.

One could ask how well a spherical antenna can determine the direction of a source. The Schenberg antenna operating at $T \sim 20 m K$, for example, may have an angular resolution of $\theta \approx 2^{\circ}$ [4]. With the help of any electromagnetic data it is possible to locate and identify a given source.

When we take such a small window (2.8-3.4 kHz) to such a giant garden named the Universe, we are sure that we are losing a great amount of information. However, if we are able to focus on good accuracy to this small region, we can also expect to see all the magnificence of it. This is the case we are proposing in this work.

We analyze the case of a possible future detection made by these antennas in order to obtain information on its GW sources. In the case of BHs, a direct detection of these mysterious objects is already of great relevance, but we are specially concerned to the information we can extract from a NS detection. If values of mass and radius can be extracted with some accuracy, we could get additional information about the nuclear matter equation of state 5]. The still obscure characteristics of nuclear matter, e.g., the values of compression modulus or nucleon effective mass could be clarified. We could also get information on the strange quark star candidates, strange quark matter equation of state and phase transition $[6]$.

A relevant and actual issue in astrophysics refers to the existence and detection of strange quark stars. Some objects have been recently detected 
and issued as possible candidates to be stars formed by deconfined quark matter. This is the case of EXO 0748-676, 4U 1700+24, 1E1207-5209 and RX J1856.5-3574 7, 8]. We do not intend, in this work, to discuss the real constitution of these stars, we just intend to show how a GW detection could identify a star as a strange star.

This paper is organized as follows. In section 2 we briefly review the $\mathrm{BH}$ and NS pulsating modes, in section 3 we discuss the detectability of NS modes by the Brazilian antenna, in section 4 we consider the constraints a putative detection by this very antenna would impose on a class of equation of states, finally in section 5 we present the final remarks.

\section{Black Holes and Neutron Stars}

NSs and BHs are certainly the most important sources of GWs, which can be generated by a host of pulsating modes of these stars.

While BHs oscillate due to its spacetime structure perturbation, NSs may also oscillate due to its fluid perturbation. This difference implies that a NS may oscillate in many different modes and have a richer spectrum of oscillations.

An excited $\mathrm{BH}$ may emit a great amount of GWs during its ringdown phase. The ringdown waveform may be completely described by the BH's mass and angular momentum.

Although NSs possess many pulsation modes only a few of them, however, is of relevance for GW detection. Concerning the GW point of view the most important modes for the NSs are the fundamental (f) mode of fluid oscillation, the first few pressure (p) modes and the first GW (w) mode [9].

It is worth noting that different types of initial perturbations are more convenient to excite each of these modes [10]. The problem of initial value in numerical relativity is one of the most challenging problems to be solved in the present. However, there is a currently belief that the fundamental mode (f-mode) may carry the greatest part of the released energy, because the fluid parameters undergo the largest changes, while the pressure (p-mode) and curvature (w-mode) would carry smaller fraction of the energy.

It is worth mentioning that the r-mode can also be, under certain circumstances, a very important source of GWs for the NSs [1].

An important question is how pulsating modes are excited in the NSs, which are of our concern here. There are many scenarios that could lead to 
significant asymmetries. Supernova explosions are expected to form wildly pulsating NSs that emit GWs. A pessimistic estimate for the energy radiated as GWs indicates a total release equivalent to $<10^{-6} M_{\odot}$. An optimistic estimate, when the NS is formed, for example, from strongly non-spherical collapse, suggests a release equivalent to $10^{-2}-10^{-1} M_{\odot}$. (see, e.g., Refs. 11, 5, 6, 12, 13, 14.

Another possible excitation mechanism for NS pulsation is a starquake, which can be associated with a pulsar glitch. The energy released in this process may be of the order of the maximum mechanical energy stored in the crust of the NSs, which is estimated to be of $10^{-9}-10^{-7} M_{\odot}$ [15, 16]. In a recent study [17] we consider the detectability of f-mode NSs in this scenario by the Brazilian antenna Schenberg. We showed that several events every year could be detectable by this antenna.

Soft gamma ray repeaters could be thought as starquakes occuring in magnetars. This process would be responsible for an energy release, in the form of GWs, about to $E \sim 10^{-6} M_{\odot} c^{2}$, being detectable if it is in our own galaxy [18].

Orbiting or falling masses into NSs have been recently studied as models for coalescing or acreting binaries [12]. Stellar oscillations being excited by the tidal fields of the two stars, for example.

Core quakes due to phase transitions in the inner shells of the NS could be able to excite pulsation modes, releasing about $E \sim 10^{-2}-10^{-1} M_{\odot} c^{2}$ in the form of GWs, and could be detected up to the Virgo cluster [6, 14]. Similarly, the transformation of a NS into a strange star is likely to induce pulsations.

Also, in ref. [19], the rate of phase transition inside NSs has been estimated to be about $10^{-6}$ events per year and per galaxy. If in fact a detection could be possible up to the Virgo cluster, this would represent a possible source of GWs.

Excitation of f-mode in LMXBs is also possible, and in this case the GW could well be recurrently produced, since the NS is continuously receiving matter from its companion star. In a recent paper de Araujo etal [20] study such a case.

Since the f-mode unstable NSs in LMXBs could well be a recurrent source of GWs, this implies that a non negligible event rate could occur in this case. A rough estimate, based on the event rate of superbursts in LMXBs, indicates that two events every year could be detected if a sensitivity for burst sources of $h_{s} \sim 10^{-23}$ could be achieved, and also if the efficiency of generation of 
GWs could be of $\varepsilon_{G W} \sim 10^{-11}$.

Finally, it is worth mentioning that Kokkotas etal [21] shows that detecting the f-mode, the EOS, the mass and the radius of the NSs will be strongly constrained. The reader should appreciate the reading of this paper by Kokkotas etal who show in detail how these above mentioned astrophysical information is obtained from the GW data.

In the following subsection we briefly review the main properties of the NSs pulsating modes.

\section{1 f- and p-modes}

The pressure modes are the overtones of the fundamental mode. The fundamental mode can be described by the density distribution inside the star, while the p-mode restoration force is the pressure. The f-mode, for a typical NS, may appear with a GW frequency of $\omega \sim 2.8 \mathrm{kHz}$ and damping time of $\tau \sim 0.1 \mathrm{~s}$ while the first p-mode appears with $\omega \sim 6 \mathrm{kHz}$ and $\tau \sim 0.6 \mathrm{~s}$ [1.

The fraction of energy released by each mode of oscillation is still an open problem. Only full 3D relativistic numerical simulations, with appropriate initial conditions, could give us considerable new information about this issue. Even though, it is a common belief, as already mentioned, that the fundamental mode is more likely to be excited than the pressure modes. It would be required tens to hundred times more powerful events to make the detection of a pressure mode comparable to the fundamental one [1]. Higher pressure modes shall not contribute considerably to the GW spectrum.

At the actual stage of GW detectors it would be required an effective amplitude about $h_{\text {eff }} \sim 10^{-21}$ to claim for a detection. The effective amplitude for an f-mode, described by Kokkotas [1] is given by

$$
h_{e f f} \sim 2.2 \times 10^{-21}\left(\frac{E}{10^{-6} M_{\odot} c^{2}}\right)^{1 / 2}\left(\frac{2 k H z}{f}\right)^{1 / 2}\left(\frac{50 k p c}{r}\right),
$$

where $E$ is the energy released in the mode, $f$ is the frequency in $k H z$ and $r$ is the distance to the source in $k p c$.

In Ref. 22] the authors have calculated the properties of the oscillation modes using a wide sample of equations of state. As the main results, they have obtained empirical formulae for, among others, the frequency of the fand first p-mode 


$$
\begin{gathered}
\nu_{f}=0.79( \pm 0.09)+33( \pm 2) \sqrt{\frac{M}{R^{3}}}, \\
\nu_{p}=\frac{1}{M}\left[-1.5( \pm 0.8)+79( \pm 4) \frac{M}{R}\right] .
\end{gathered}
$$

where the masses and radii are given in $\mathrm{km}$, while $\nu_{f}$ and $\nu_{p}$ are given in $\mathrm{kHz}$.

Inverting the problem and considering the figures to the Schenberg (3.0$3.4 \mathrm{kHz})$ and Mini-GRAIL $(2.8-3.0 \mathrm{kHz})$ detectors bandwidths, one sees the kind of information it is possible to obtain by using the above equations.

Assuming a putative detection of a standard $1.4 M_{\odot} \mathrm{NS}$, a very small radius, namely, $R \sim 7.3 \mathrm{~km}$, would be inferred, which is too small for a standard NS.

As another example, if a solar mass f-mode unstable NS is detected, the radius of the star would be constrained to $R=6.58 \pm 0.79 \mathrm{~km}$. Given that this radius is too small for a NS, it would be certainly a strange quark star.

Note that a NS of $M \sim 1.0(1.4) M_{\odot}$ with $R \sim 14 \mathrm{~km}$ would emit waves in much lower frequencies, $\nu_{f} \sim 1.5(1.7) k H z$.

Also, if a detection is done and identified as a standard $1.4 M_{\odot} \mathrm{NS}$ p-mode unstable, a too large radius would be inferred, namely, $R \sim 20 \mathrm{~km}$, which is inconsistent with any NS model.

If the detection points to a putative half solar mass NS p-mode unstable, it would lead to a radius of $R=16.26 \pm 4.75 \mathrm{~km}$. This result could hardly, by itself, rule out any of the present equations of state, specially considering that the p-mode damping is very dependent on the stellar model and cannot be well fitted as in the case of the f-mode (see Ref. 22 for details). Due to its frequency bandwidth, the Schenberg detector could not see NSs far from this mass range.

NSs have a very rich spectrum of mechanical oscillations, however, other modes, like w-, g- and r-modes, are not considered here because their frequencies lie on a very different range and cannot be detected by the Brazilian and the Dutch detectors, considered in this work.

\section{$3 \quad$ Schenberg Bandwidth}

One of the main purposes of this work is to establish the main properties of the sources when the Schenberg's bandwidth $(3.0-3.4 \mathrm{kHz})$ is considered. 
From the above discussion the natural candidates for Schenberg are BHs and NSs.

In the present study, we are particulary concerned with the determination of the equation of state of NSs. A relevant question is how to know, in a putative detection by Schenberg, if the source is a NS or a BH. The basic way to distinguish them can be through the damping times of their oscillating modes. The damping time for the quasi-normal modes of BHs is orders of magnitude shorter than the f- and p- modes of NSs.

There is a simple relation correlating a Schwarzschild BH mass to the frequency of its fundamental (quadrupole) quasi-normal mode (see, e.g., Ref. 1])

$$
\omega=12.07 \mathrm{kHz}\left(\frac{M_{\odot}}{M}\right)
$$

where $\mathrm{M}$ is the mass in $M_{\odot}$. This relation implies that the Schenberg antenna will only see BHs if their masses are about $3.5-4.0 M_{\odot}$. It is worth mentioning that if rotation is considered the above mass range could be extended. A BH of $9 M_{\odot}$ at maximum rotating velocity could in principle be seen by Schenberg [23].

The damping time of this mode is given by [1]

$$
\tau=0.05 \mathrm{~ms}\left(\frac{M}{M_{\odot}}\right) ;
$$

which for BHs with masses $3.5 \leq M \leq 4.0 M_{\odot}$ gives $\simeq 0.2 \mathrm{~ms}$.

The same procedure is now applied to identify $\mathrm{f}$ - or p-modes in the Schenberg antenna. A NS f-mode with frequency about $3.0 \mathrm{kHz}$ presents a damping time much larger then those presented by a $\mathrm{BH}$, being of the order of $100 \mathrm{~ms}$. This is also the case for the first p-mode, which would have damping times greater then a few seconds. So, the differences of a BH ringdown and a NS f- and p-modes are easily identified by their corresponding damping times.

Even though Schenberg could not determine the damping times within low errors, such a great difference between these three modes could enable us to determine the mode that would excite the sphere.

As a restricted bandwidth implied in a corresponding restriction of $\mathrm{BH}$ candidates due to their masses, it shall also imply in a restriction of NSs candidates. In order to do a model-independent analysis, we have applied the Schenberg bandwidth into the empirical relations obtained by Benhar 
etal [22. These relations determine the wave frequency and damping time when the stellar mass and radius is known.

We have followed in the opposite direction and determined the NS masses and radii that would emit f- or p-mode waves in the detector Schenberg bandwidth. The main results of this analysis are synthesized in figure 11, where we have plotted the regions that correspond to NSs whose GW lies in Schenberg frequency band. The NS mass-radius relation determines the candidates for a detection by the Schenberg antenna.

Considering, in equation 1, a bandwidth of $3.0-3.4 \mathrm{kHz}$ we obtain the mass-radius relation represented by the lower shaded region in figure 1 :

$$
R=\left(\frac{33( \pm 2)}{3.2( \pm 0.2)-0.79( \pm 0.09)}\right)^{2 / 3} M^{1 / 3} .
$$

The upper shaded region is obtained through the same procedure, using the empirical formula for the first pressure mode (equation 2):

$$
R=\frac{79( \pm 4) M}{3.2( \pm 0.2) M+1.5( \pm 0.8)},
$$

where, in either of above equations, the mass and radius are given in $\mathrm{km}$.

We also plotted in figure 1 a couple of equations of state such as the Taurines [24] and non-linear (NL) 6] models for nuclear matter, the MIT bag model 25] and the Chromo-Dieletric model [26] for quark matter, which we consider in detail in the next section.

If a f-mode is identified in the detector by its damping time, the most probable source, considering the results of figure 1, would correspond to a very compact star with radius smaller than $10 \mathrm{~km}$. The models that fulfill this condition are models of strange quark stars. NSs with higher masses can hardly account such a small radius. However, if such a detection is done, it would lead to important constraints on the NS matter equation of state, since only a few models and free parameters sets can account for NSs with $M \approx 2 M_{\odot}$ and $R \approx 10 \mathrm{~km}$ at the same time. On the other hand, pressure modes would only be expected to come from low-mass NSs with very large radii.

From the previous analysis, and considering the fact that low-mass NSs do not appear in a great number in our galaxy, strange stars are the most probable sources of f-mode waves to be detected by the Schenberg antenna. Most of the modern NS models cannot account for the small radius values 
imposed by the $3.0-3.4 \mathrm{kHz}$ bandwidth. One can also consider the fact that RX J1856-3754 8 can, in fact, be a strange quark star, and its inferred values of mass and radius would imply in a f-mode eigenfrequency about that considered by the Schenberg bandwidth.

It is worth noting that the constraint given by the Mini-GRAIL detector is lighter (see 2), since some other EOSs, of those considered in the present paper, would contribute to its bandwidth.

Even though the Schenberg spherical antenna cannot determine the properties of damping time with low errors, we applied the empirical relations obtained by Benhar etal, as we have done before, to describe its properties, considering the NSs in which the f-mode frequency lies between $3.0-3.4 \mathrm{kHz}$. In Ref. 22] the authors found an empirical relation for the f-mode damping time described by

$$
\tau_{f}=\frac{R^{4}}{c M^{3}}\left[(8.7 \pm 0.2) \cdot 10^{-2}+(-0.271 \pm 0.009) \frac{M}{R}\right]^{-1}
$$

where $R$ is the radius and $M$ is the gravitational mass, both in units of $k m$ and $c$ is the speed of light, $3 \cdot 10^{5} \mathrm{~km} / \mathrm{s}$. In addition to the frequency equation, $\mathrm{Eq}$ 2, that gives rise to the detectable region in the mass-radius diagram, Eq[6, we obtained the diagram drawn in Fig[2. This diagram shows that, in the Schenberg detectable region, the signal obtained from NSs with very high masses, $M>1.8 M_{\odot}$, would have a damping time of, at least, $0.2 s$, while very low mass NSs, $M<0.5 M_{\odot}$ would have damping times $\tau>0.1 s$. NSs with masses around $1 M_{\odot}$ would have a wide range of possible damping times, $0.06-0.10 s$.

At this point the relevant questions are: what about if a detection is done? What kind of information we can get from it? These are the main questions of our study. We have already seen how we could determine some of the source properties, such as the mass and radius, and now we need to consider some discussion concerning the nuclear matter equation of state to find out what kind of information all of this together could give. We deal with these issues in the next section.

\section{Nuclear Matter Equation of State}

We have seen that a possible GW detection by the Schenberg antenna would impose severe constraints in the properties of its sources, e.g., the masses and 


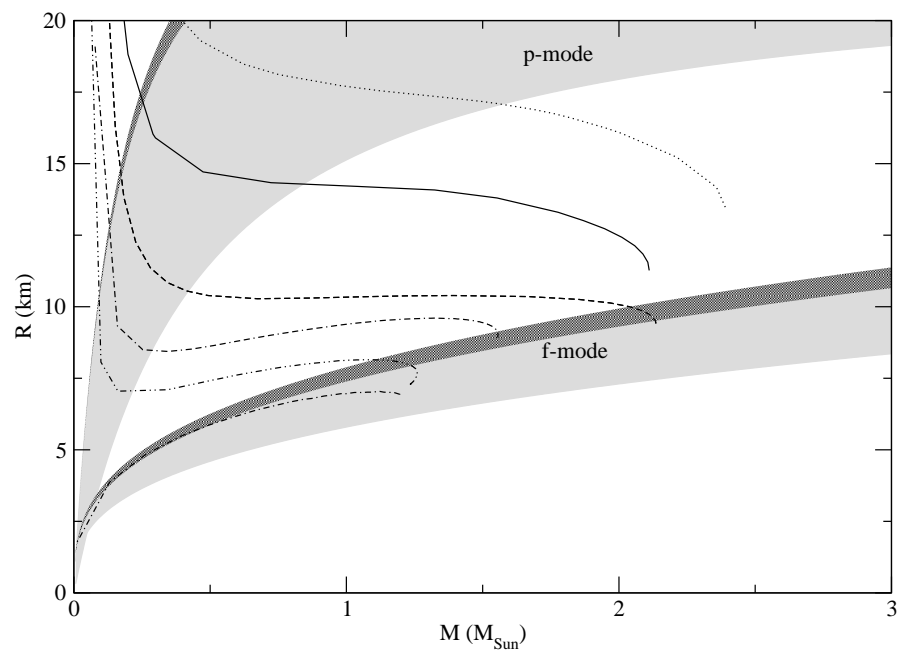

Figure 1: Mass-radius relation for the Benhar etal empirical relation and for different EOSs. The light shaded regions represent the empirical relations for the f- (lower) and first p-mode (upper) for the Schenberg bandwidth while the dark ones describe the additional contribution due to the MiniGRAIL bandwidth. The results are compared, for the NL model, to the normal static (solid line) and (maximum) rotating NS (dotted line) and the Taurines model with $\mathrm{K}=220 \mathrm{MeV}$ (dashed line). Static strange quark stars for the MIT bag model are plotted with $B=60 \mathrm{MeV} / \mathrm{fm}^{3}$ (dot-double dashed line) and $B=100 \mathrm{MeV} / \mathrm{fm}^{3}$ (dash-double dotted line) and Chromo-dieletric model (dot-dashed line). 


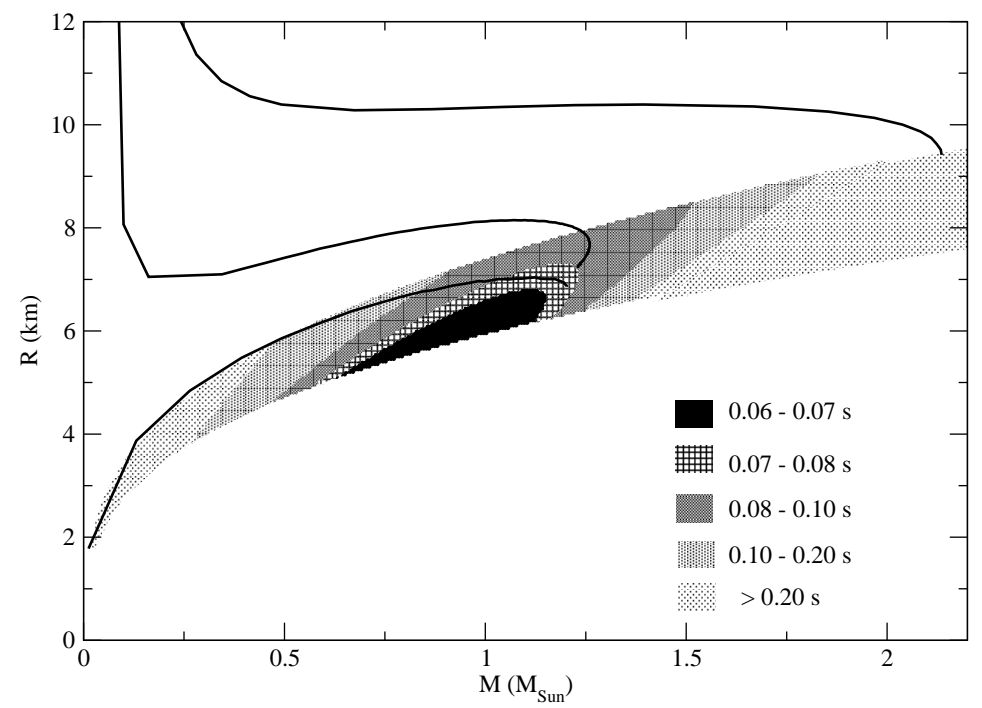

Figure 2: Mass-radius relation for the Benhar etal empirical relation. The shaded regions represent the empirical relations for the f-mode with different damping times. 
radii of the NSs. Once we have considered these constraints, we can invert the problem and verify its relevance in the nuclear matter equation of state parameters. Some attempts have already been done in this direction [27] but one of the purposes of this work is to study the information we can get from the Brazilian detector.

The global properties of mass and radius of compact stars are directly related to its equation of state. In the previous section we have developed our analysis in a model-independent level. Here, we go a step further and, using a specific model for NS matter, we analyze the consequences of a detection. In particular, we consider the model developed by Taurines etal 24] for a new class of parameterized field-theoretical model (see figure 3) described by the following lagrangian density

$$
\begin{aligned}
\mathcal{L}= & \sum_{B} \bar{\psi}_{B}\left(i \gamma_{\mu}\left(\partial^{\mu}-g_{\omega B}^{\star} \omega^{\mu}\right)-\left(M_{B}-g_{\sigma B}^{\star} \sigma\right)-\left[\frac{1}{2} g_{\varrho B}^{\star} \boldsymbol{\tau} \cdot \boldsymbol{\varrho}^{\mu}\right]\right) \psi_{B} \\
& +\frac{1}{2}\left(\partial_{\mu} \sigma \partial^{\mu} \sigma-m_{\sigma}^{2} \sigma^{2}\right)-\frac{1}{4} \omega_{\mu \nu} \omega^{\mu \nu}+\frac{1}{2} m_{\omega}^{2} \omega_{\mu} \omega^{\mu} \\
& -\frac{1}{4} \boldsymbol{\varrho}_{\mu \nu} \cdot \boldsymbol{\varrho}^{\mu \nu}+\frac{1}{2} m_{\varrho}^{2} \boldsymbol{\varrho}_{\mu} \cdot \boldsymbol{\varrho}^{\mu}+\sum_{l} \bar{\psi}_{l}\left[i \gamma_{\mu} \partial^{\mu}-M_{l}\right] \psi_{l}
\end{aligned}
$$

where the baryon field $\psi_{B}$ is summed over the whole baryon octet and coupled to the scalar and vector fields $\sigma, \omega$ and $\varrho$ through the parametrized coupling constants $g_{\sigma B}^{\star}, g_{\omega B}^{\star}, g_{\varrho B}^{\star}$. The free lepton fields $\psi_{\lambda}$ contributes to the eletrical equilibrium in the NS matter. The masses of the baryons, mesons and leptons are represented by $M_{B}, m_{\sigma, \omega, \varrho}$ and $m_{\lambda}$, respectively.

Using a parameterization, $\lambda$, for the baryon-meson coupling constants,

$$
g_{\sigma}^{\star} \bar{\psi} \sigma \psi=\frac{g_{\sigma} \sigma}{\left(1+\frac{g_{\sigma} \sigma}{\lambda M}\right)^{\lambda}} \bar{\psi} \psi
$$

this model describes a wide range of NS parameters, such as a maximum mass ranging from (very low values) $M=0.66 M_{\odot}$ up to $M=2.77 M_{\odot}$, and the corresponding radii that vary in the range of $8<R<13.17 \mathrm{~km}$. Each pair in the mass-radius relation is associated with a different parametrization of the equation of state (see Ref. 24] for further details). Of course, each value of such parametrization represents different values of nuclear matter properties.

The study of nuclear matter properties is still a very open problem. We focuse here on the problem of the determination of the compression modulus, 


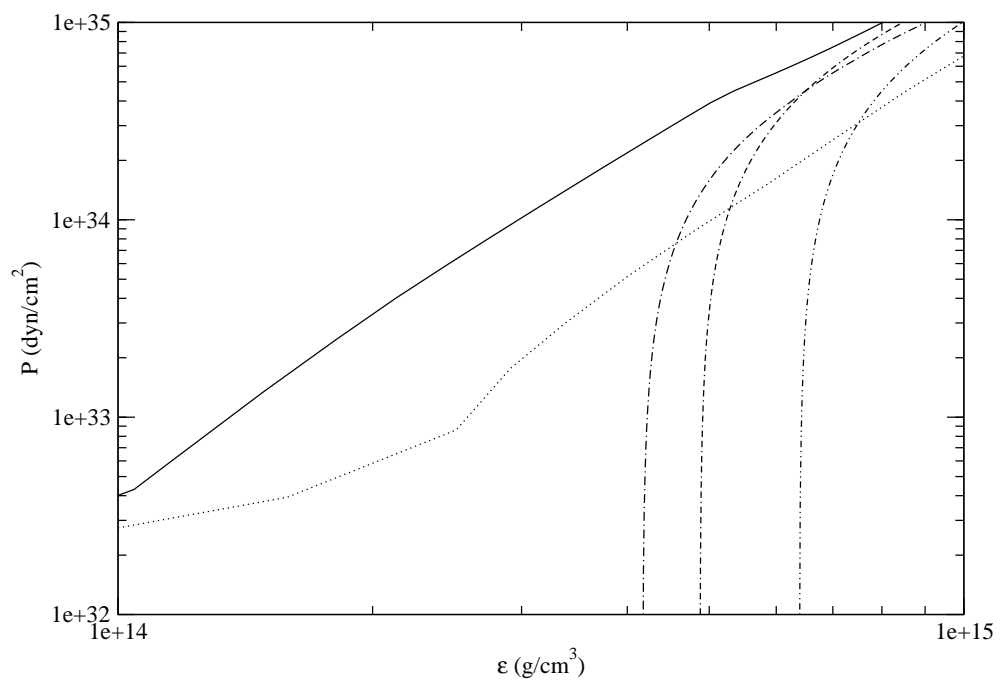

Figure 3: Equations of State for normal nuclear matter, using the Taurines model (solid line) and NL model (dotted line), and for quark matter using the Chromo-dieletric model (dot-dashed line) and MIT bag model with $B=60 \mathrm{MeV} / \mathrm{fm}^{3}$ (dash-double dotted line) and with $B=100 \mathrm{MeV} / \mathrm{fm}^{3}$ (dot-double dashed line). 


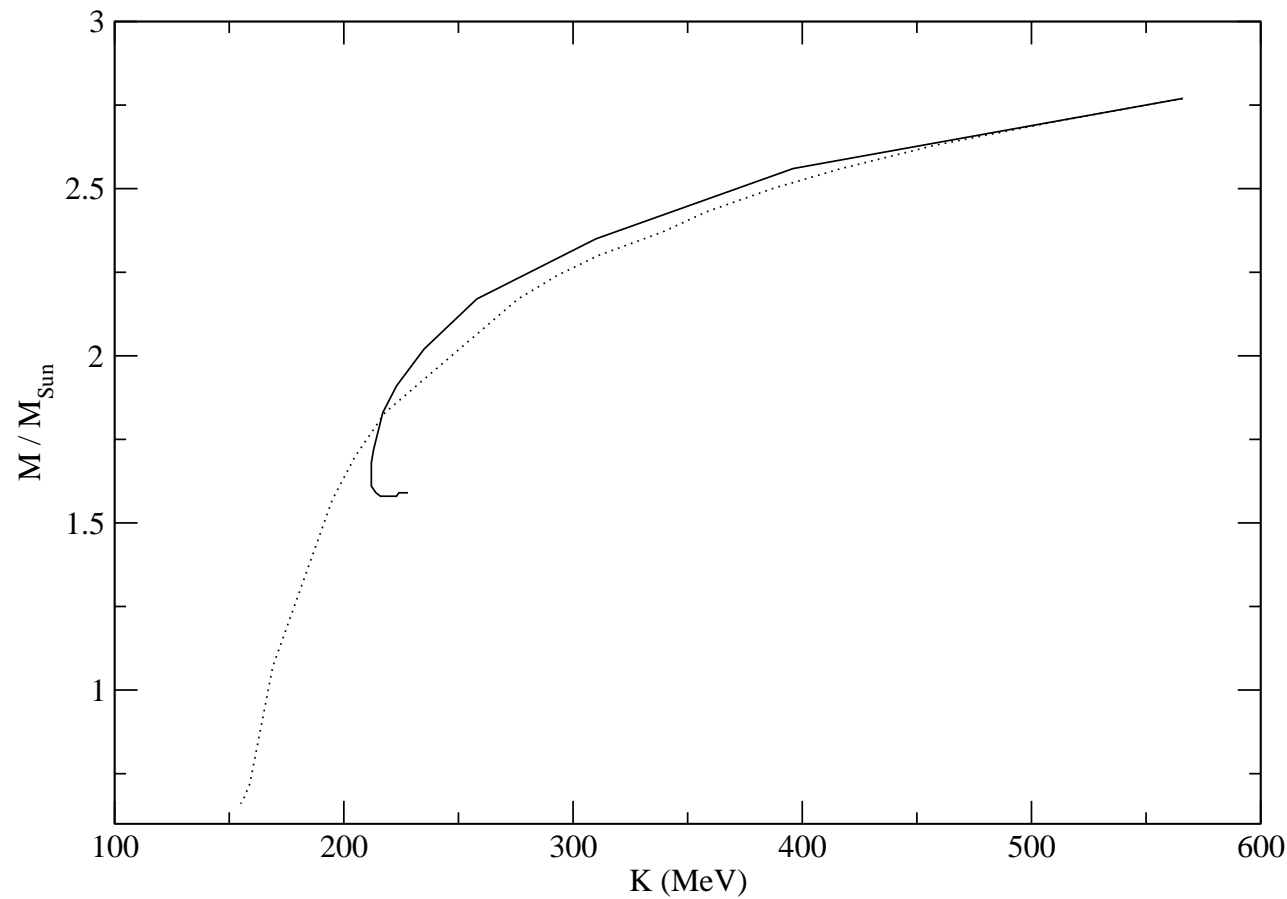

Figure 4: The NS maximum masses versus the compression modulus, for different parametrization in the Taurines etal models. The solid line represents a parametrization in the scalar mesons while the dotted line describes a parametrization in both scalar and vector contributions of the nuclear force.

$K$. The compression modulus defines the curvature of the equation of state or, in a few words, is related to the capability of matter to be compressed. One can see in figure 4 that a maximum mass of NSs is obtained for large values of the compression modulus. The definition of $K$ reads

$$
K=9\left[\rho^{2} \frac{d^{2}(\epsilon / \rho)}{d \rho^{2}}\right]_{\rho=\rho_{0}}
$$

where $\epsilon$ is the energy density, $\rho$ is the baryon density and $\rho_{0}$ the baryon saturation density. Actually, the acceptable values for the compression modulus are constrained between $200<K<300 \mathrm{MeV}$ [28, 29].

An important constraint to the equations of state is to describe a family of NSs with a maximum mass greater than the value obtained by the most 
massive known pulsar. The PSR J0751+1807 has a mass $M=2.1 \pm 0.2 M_{\odot}$ 30. This condition has to be fulfilled by the set of parameters chosen to describe the NS model. This first condition already constrains the value of compression modulus to $K \geq 220 \mathrm{MeV}$ when this model is considered. Smaller values of $\mathrm{K}$ would lead to masses smaller than $1.9 M_{\odot}$ disagreeing with the measures of PSR J0751+1807 mass.

Assuming the detection of a f-mode, associated with a NS composed of nuclear matter, which we have seen as very unexpected, this would imply a very compact $\mathrm{NS}$, with high masses $\left(>1.8 M_{\odot}\right)$ occupying a small radius, namely, 9-10 km. As the maximum mass star has the smallest radius (see figure 1), we look for a set of parameters which leads to the smallest radius for the maximum mass star. Stellar models on which the maximum mass has a radius $R \leq 10 \mathrm{~km}$ have compression modulus $215<K<225 \mathrm{MeV}$. Stellar models with masses smaller then $2.5 M_{\odot}$ and radii greater then $10 \mathrm{~km}$ cannot be detected by the Schenberg antenna, so, a putative detection would be responsible for the most important constraint on the values of $K$ even done by any earth-based nuclear experiment, namely, $220<K<225 \mathrm{MeV}$.

It was already proposed the existence of low-mass NSs in LMXBs and HMXBs (see, e.g., Refs. [31, 32]). The detection and identification of a p-mode wave would confirm this existence. It is worth mentioning that, as a spherical antenna can be used to identify the direction of the source, the detection could also confirm that low-mass NSs are, in fact, related to the LMXBs and HMXBs.

Another scenario to be discussed concerns the detection of an f-mode when the source is a strange quark star. The models used in this analysis are the MIT bag model [25] and the chromo-dielectric model [26]. The detection of an f-mode wave coming from a strange star would impose new constraints on the values of the bag constant $\mathrm{B}$, the strange quark mass and the perturbative constant $\alpha_{c}$.

The MIT bag model describes, as its own name suggests, that baryons are composed by three quarks confined inside a bag. The effects of pressure difference in the interior and exterior regions of the bag are summarized in the bag constant. The thermodynamical potential of quarks in the MIT bag model is described by

$$
\Omega=\sum_{q=u, d, s} \frac{-1}{4 \pi^{2}}\left[\mu_{q} k_{F q}\left(\mu_{q}^{2}-\frac{5}{2} m_{q}^{2}\right)+\frac{3}{2} m_{q}^{4} \ln \left(\frac{\mu_{q}+k_{F q}}{m_{q}}\right)\right]
$$




$$
\begin{aligned}
& +\frac{2 \alpha_{c}}{4 \pi^{3}}\left[3\left[\mu_{q} k_{F q}-m_{q}^{2}-m_{q}^{2} \ln \left(\frac{\mu_{q}+k_{F q}}{m_{q}}\right)\right]^{2}\right. \\
& -2 k_{F_{q}}^{4}-3 m_{q}^{4} \ln ^{2}\left(\frac{m_{q}}{\mu_{q}}\right)+6 \ln \left(\frac{\rho_{r}}{\mu_{q}}\right)\left[\mu_{q} k_{F q} m_{q}^{2}\right. \\
& \left.\left.-m_{q}^{4} \ln \left(\frac{\mu_{q}+k_{F q}}{m_{q}}\right)\right]\right]+B
\end{aligned}
$$

where $q=u, d, s$ are the three quark flavors up, down and strange, $\mu_{q}$ is the chemical potential, $m_{q}$ represents the quark masses, $k_{q}$ is the Fermi momentum of the particles, $B$ is the bag constant and the terms multiplied by $\alpha_{c}$ represents the contribution of the first order perturbation in the strong interaction.

Even with considerable advances in the study of QCD (quantum chromodynamics) on the lattice, from where we expect the most reliable theoretical results and the consequent advances in collision experiments, the bag constant still presents a very wide range of possible values. In the same way, the perturbative constant, $\alpha_{c}$, the one that represents the first order correction to the strong interaction forces between quarks, also represents an open issue. Astrophysical and GW experiments may provide a short-cut to the determination of these values.

We argue that, if a GW detection is done, and identified as an f-mode wave coming from a strange quark star, the higher are the values of the bag constant, $B$, the perturbative constant, $\alpha_{c}$ and the strange quark mass, $m_{s}$, the more compact is the star and more the model fits into the region of Schenberg's sensibility curve.

The set of constants, $B=60 \mathrm{MeV} / \mathrm{fm}^{3}, \alpha_{c}=0$ and $m_{s}=150 \mathrm{MeV}$ could not describe stars that emit $3.0-3.4 \mathrm{kHz}$ waves, on the other hand, $B=100 \mathrm{MeV} / \mathrm{fm}^{3}, \alpha_{c}=0.35$ and $m_{s}=200 \mathrm{MeV}$ would perfectly fit the optimum region. Our results are in perfect agreement with those found in Ref. 27], where the higher is the value of $\mathrm{B}$, the higher is the f-mode frequency.

The same behavior appears in the color-dieletric model when we convert the parameters to obtain the corresponding values of a bag constant. These constraints in the values of the bag and perturbative constants would severely restrict the QCD parameters. If a detection of a strange star is done by the Schenberg antenna and, consequently, these values are confirmed, the hadronquark phase transition would occur in an extremely high density. Such high density would rule out the possibility of finding standard NSs with quark 
cores.

Last, but not least, we refer the reader to Fig. 2, from which we can conclude that the determination of the damping time could also contribute to constraint the parameters of the equations of state and also the star mass range.

\section{Final remarks}

We have briefly reviewed the properties of GW emission by NSs and BHs. We also have seen the main characteristics of the Brazilian and Dutch spherical antennas.

Assuming a possible detection of GWs coming from a NS in the range of $2.8-3.4 \mathrm{kHz}$, the one by Schenberg and Mini-GRAIL, we can extract information to describe the source characteristics. The identification of the source could be done by means of the wave damping time, which combined with the corresponding frequency, leads to the determination of the mass and radius of the star.

The determination of these values imposes important constraints on the nuclear and subnuclear matter equation of state, such as limiting values for the compression modulus, nucleon effective mass, bag and perturbative constants. As a matter of example, values like $K \approx 220 \mathrm{MeV}, B \approx$ $100 \mathrm{MeV} / \mathrm{fm}^{3}$ and $\alpha_{c} \approx 0.35$ would be imposed by a putative detection.

It is still important to mention that spherical antennas can identify the position of the source in the sky, with $20^{\circ}$ for the first stage of the Schenberg antenna, operating at $T \sim 4.2 K$ and about $2^{\circ}$ at mili-Kelvin temperatures [23. This property would enable us to combine GW data with those obtained by observations in the electromagnetic spectra, which together could help identifying the sources.

Last but not least, a relevant question has to do with the event rate of detectable oscillating modes of NSs within the Schenberg bandwidth. We have seen in the introduction of the present paper that there exists a host of possible mechanisms to excite the oscillating modes of NSs. Many of these mechanisms, however, present very uncertain event rates. If the f-mode is excited, for example, during the formation of the NSs, the event rate would be the one related to the rate of supernovae, which for our Galaxy amounts one event every tens of years.

In recent studies de Araujo etal [17, 20] consider the event rates associated 
with pulsar glitches and also in LMXBs. They argue that up to several event years would be possible. We refer the reader to these studies for further details.

\section{Aknoledgments}

GFM and JCNA would like to thank CNPq (grants 381682/2006-4, $303868 / 2004-0$, respectively) for financial support. We also would like to thank Flavio D'Amico for fruitful and helpful discussions concerning the X-

ray binaries. Last, but not least, we would like to thank the referees whose suggestions and criticisms greatly improve our paper.

\section{References}

[1] K. D. Kokkotas, B. G. Schmidt 1999 Living Rev. Rel. 22

[2] O. D. Aguiar et. al. 2005 CQG 22 S209

[3] A. de Waard et. al. 2005 CQG 22 S215

[4] C. A. Costa (from the Schenberg team) 2006 private communication

[5] K. D. Kokkotas, T. A. Apostolatos, N. Andersson 2001 MNRAS 320 307

[6] G. F. Marranghello, C. A. Z. Vasconcellos, J. A. de Freitas Pacheco 2002 PRD66 064027

[7] M. Bagchi, S. Ray, M. Dey, J. Dey 2006 MNRAS 368971

[8] J. J. Drake, H. L. Herman 2003 Nucl. Phys. A718 351

[9] Kokkotas K D and Schutz B F 1992 MNRAS 255119

[10] J. Ruoff, P. Laguna, J. Pullin 2001 PRD63 064019

[11] Andersson N, Kokkotas K D, Stergioulas N 1999 Astrophys. J. 516307

[12] J. A. Pons, E. Berti, L. Gualtieri, G. Miniutti, V. Ferrari 2002 PRD65 104021 
[13] J. E. Horvath 1996 IJMP D5 35

[14] L.-M. Lin, K. S. Cheng, M.-C. Chu, W.-M. Suen 2006 ApJ 639382

[15] Blaes O, Blandford R, Goldreich P, Madau P 1989 Astrophys. J 343839

[16] Mock P C and Joss P C 1998 Astrophys. J. 500374

[17] J. C. N. de Araujo, O. D. Miranda, O. D. Aguiar 2005 CQG 22 S471

[18] J. A. de Freitas Pacheco 1998 A $\& A 336397$

[19] F. Ma, B. Xie 1996 Astrophys. J. 462 L63

[20] J. C. N. de Araujo, O. D. Miranda, O. D. Aguiar 2006 J. Phys.: Conf. Ser. 321

[21] K. D. Kokkotas, T. A. Apostolatos, N. Andersson 2001 MNRAS 320 307

[22] O. Benhar, V. Ferrari, L. Gualtieri 2004 PRD70 124015

[23] C. A. Costa, O. D. Aguiar, N. S. Magalhes 2004 CQG 21 S827

[24] A. R. Taurines, C. A. Z. Vasconcellos, M. Malheiro, M. Chiapparini 2001 PRC63 065801

[25] A. Chodos, R. L. Jaffe, K. Johnson, C. B. Thorne, V. F. Weiskopf 1974 PRD9 3471

[26] M. Malheiro, M. Fiolhais, A. R. Taurines 2003 J. Phys. G29 1045

[27] O. Benhar, V. Ferrari, L. Gualtieri, S. Marassi 2006 astro-ph/0603464

[28] Blaizot, J. P. 1980 Phys. Rep. 64171

[29] Myers, W. D., Swiatecki, W. J. 1996 Nucl. Phys. A601 141

[30] D. J. Nice etal 2005 Astrophys. J. 6341242

[31] Jonker, P.G. van der Klis, M., and Groot, P. J. 2003 MNRAS 339663

[32] Orosz, J., and Kuulkers, E. 1999 MNRAS 305132 